Original Paper http://ajol.info/index.php/ijbcs http://indexmedicus.afro.who.int

\title{
Approche ethnobotanique et ethnopharmacologique des plantes utilisées dans le traitement traditionnel de l'ulcère de Buruli à Akonolinga (Cameroun)
}

\author{
Hervé Narcisse BAYAGA ${ }^{1,2}$, Nicole Marie GUEDJE ${ }^{1 *}$ et Elvire Hortense BIYE ${ }^{2}$ \\ ${ }^{1}$ Département de Pharmacognosie et de Chimie Pharmaceutique, Faculté de Médecine et des Sciences \\ Biomédicales, Université de Yaoundé 1. B.P.: 1364 Yaoundé, Cameroun. \\ ${ }^{2}$ Département de Biologie et Physiologie Végétales, Faculté des Sciences, Université de Yaoundé 1. B.P.: 812 \\ Yaoundé, Cameroun. \\ *Auteur correspondant ; E-mail: manigue@hotmail.com; Tél.: +237676075355
}

\section{REMERCIEMENTS} Cameroun.

Ce travail a été rendu possible grâce à l'allocation du Ministère de l'Enseignement Supérieur du

\section{RESUME}

Peu de données sont disponibles sur les plantes utilisées dans le traitement traditionnel de l'Ulcère de Buruli au Cameroun. Cette étude avait pour objectif l'identification des espèces et la caractérisation des recettes anti-ulcères de Buruli dans l'arrondissement d'Akonolinga. L'enquête ethnopharmacologique a permis d'identifier 25 plantes utilisées contre l'Ulcère de Buruli. L'écorce des tiges $(41,1 \%)$, les tiges $(26,8 \%)$ et les feuilles (25\%) étaient les organes les plus utilisés. La décoction $(64,3 \%)$ et le pilage $(23,2 \%)$, étaient les modes de préparation prépondérants, s'administrant par voie externe, principalement par massage et désinfection de la lésion $(64,3 \%)$ et en cataplasme (14,3\%). L'analyse des citations d'usages, des similitudes d'emplois et convergence d'usage a montré que Musa parasidiaca, Mitracarpus villosus, Aframomum melegueta, Elaeis guineensis et Spathodea campanulata faisaient partie des espèces aux convergences d'usages remarquables entre différentes régions géographiques, tandis que sept autres, Petersianthus macrocarpus, Momordica cabraei, Cassia spectabilis, Citrus medica, Terminalia superba, Ceiba pentandra et Ipomoea aquatica, constituaient l'autre groupe d'espèces les plus significatives. En mettant en évidence ces douze espèces d'intérêt, cette étude a une applicabilité en innovation thérapeutique, par le raisonnement ethnopharmacologique, en concourant à la sélection des espèces et à l'orientation des évaluations chimiques, pharmacologiques ou cliniques prioritaires conduisant au développement de phytomédicaments dont l'Afrique a besoin pour ses pathologies endémiques.

(C) 2017 International Formulae Group. All rights reserved.

Mots clés : Ulcère de Buruli, recettes traditionnelles, espèces végétales d'intérêt, raisonnement ethnopharmacologique.

\section{Ethnopharmacological and ethnobotanical approach of medicinal plants used in the traditional treatment of Buruli ulcer in Akonolinga (Cameroon)}

\section{ABSTRACT}

Few data are available concerning plants used in the traditional treatment of Buruli ulcer in Cameroon. This study aimed to identify species and characterize anti-Buruli ulcer recipes in the Akonolinga district. The 
ethnopharmacological survey has enabled to identify 25 plant species used against Buruli ulcer. The plant parts mostly used were stem bark $(41.1 \%)$, stem $(26.8 \%)$ and leaves $(25 \%)$. Decoction $(64.3 \%)$ and pounding $(23.2 \%)$, the most dominant preparing method, were administrated externally, mostly by massage and disinfection (64.3\%) and by local poultice application (14.3\%). The analysis of relative frequency of citation, as well as the search of similarity and convergence of use, have shown that Musa parasidiaca, Mitracarpus villosus, Aframomum melegueta, Elaeis guineensis and Spathodea campanulata were among species exhibiting remarkable convergence of use between different geographical regions; while seven others, Petersianthus macrocarpus, Momordica cabraei, Cassia spectabilis, Citrus medica, Terminalia superba, Ceiba pentandra and Ipomoea aquatica, formed another most significant species group. In highlighting those 12 species of interest, this study has direct bearing in drug design and innovation by the ethnopharmacological reasoning, thereby contributing to species selection and direction for prior chemical, pharmacological and clinical assessments leading to plant-based drug development that Africa needed for its pandemic pathologies. (C) 2017 International Formulae Group. All rights reserved.

Keywords: Buruli ulcer, traditional recipes, plant species of interest, ethnopharmacological reasoning.

\section{INTRODUCTION}

Les populations africaines sont confrontées à l'émergence ou la ré-émergence de pathologies infectieuses dont le traitement et le suivi constituent, pour elles, un problème socio-économique supplémentaire. Aux premiers rangs de ces pathologies, se classe l'ulcère de Buruli (UB), infection cutanée due à une mycobactérie, Mycobacterium ulcerans, présente dans l'environnement (Bratschi et al., 2013; Aubry et Gaüzère, 2015), qui est la mycobactériose la plus fréquemment rencontrée chez l'homme actuellement après la tuberculose et la lèpre. Cette pathologie sévit dans les zones marécageuses des régions intertropicales, tropicales et subtropicales d'Afrique, d'Amérique latine, d'Asie, d'Océanie et du Pacifique occidental. Ses foyers sont géographiquement presque toujours circonscrits autour d'un écosystème aquatique, notamment les fleuves, lacs artificiels ou naturels, zones marécageuses, systèmes d'irrigation (Yemoa et al., 2008; Aubry et Gaüzère, 2015).

D'après Aubry et Gaüzère (2015), environ 5000 nouveaux cas sont répertoriés chaque année, mais beaucoup de cas ne sont pas diagnostiqués, car la maladie reste mal connue et les personnes touchées proviennent principalement des communautés pauvres et rurales. L'UB est hyperendémique en Afrique de l'Ouest et en Afrique Centrale avec une prévalence variant entre 849 cas au Congo et 11517 cas en Côte d'Ivoire. Les cas d'UB diagnostiqués au Cameroun proviennent, pour la plupart, des plaines aux nombreux marécages côtoyant le fleuve Nyong, précisément dans l'arrondissement d'Akonolinga, située dans le département du Nyong et Mfoumou et dans le district de Bankim (Pouillot et al., 2007; Bratschi et al., 2013; Andreoli et al., 2015). Cette infection, n'entrainnant que peu de décès, est cependant à l'origine d'importantes incapacités fonctionnelles car, elle conduit souvent à une destruction étendue de la peau et des tissus mous, avec formation d'ulcérations importantes généralement sur les membres. Cette atteinte élective, surtout chez des enfants, crée un double problème social de déscolarisation et de réinsertion.

Actuellement, le traitement de l'UB associant une bi-antibiothérapie à tous les stades de la maladie pendant 8 semaines entraîne la guérison des lésions précoces, une stabilisation de la maladie ou une régression des lésions permettant une exérèse chirurgicale moins délabrante (Chauty et al., 2007; Sarfo et al., 2010; Aubry et Gaüzère, 2015). Cependant, de nombreux effets 
secondaires de cette bi-thérapie ont été récensés, surtout chez les enfants, avec notamment, des atteintes hépatiques, rénales, auditives et vestibulaires. Par ailleurs, le traitement chirurgical, en combinaison ou non à la bi-thérapie, présente des résultats satisfaisants, mais aussi d'autres contraintes parmi lesquels des effets secondaires incommodes, le coût élevé et la longue durée d'hospitalisation et/ou du traitement pour de nombreux malades provenant en majorité des zones rurales; de plus, ce traitement paraît pourvoyeur de mutilations diverses, voire des amputations (Jonshon et al., 2004; Houngbédji et al., 2011; Zhang et al., 2013).

C'est ainsi que de nombreux malades ont recours, très souvent, et en première intention, à la médecine traditionnelle et aux plantes pour traiter cette pathologie (Johnson et al., 2004; Renzaho et al., 2007; Porten et al., 2009; Yemoa et al., 2008, 2015). Des travaux ont été entrepris pour mieux étudier ces plantes et thérapies traditionnelles afin de disposer des informations par rapport à l'efficacité et/ou l'innocuité de ces dernières. Plusieurs espèces de plantes médicinales traditionnellement utilisées pour traiter l'UB ont été identifiées, leur composition chimique définie et leurs activités antibactériennes sur Mycobacterium ulcerans, ainsi que leur toxicité déterminées en Afrique de l'Ouest de manière globale (Tsouh Fokou et al., 2015, 2016), et plus spécifiquement au Benin (Yemoa et al., 2008, 2011, 2015), au Ghana (Addo et al., 2007), en Côte d'Ivoire (Coulibaly et al., 2011). Au Cameroun, les travaux de Pouillot et al. (2007), Landier et al. (2011) ont identifié les facteurs de risque de l'UB, ainsi que les facteurs protecteurs contre cette pathologie au nombre desquels l'utilisation traditionnelle des feuilles (d'espèces non déterminées) pour traiter les blessures et plaies. Les travaux de Porten et al. (2009) ont estimé que la prévalence de cette pathologie dans le district de santé
d'Akonolinga était de $0,47 \%$ pour un total de 105 sujets étudiés. Andreoli et al. (2015) ont rapporté, à propos d'une étude de cas d'un patient à Bankim ayant refusé l'antibiothérapie telle que recommandée par l'OMS, que les lésions ulcéreuses ont complètement regressé après nettoyage et application par le père, d'un traitement traditionnel à base de l'écorce de deux espèces, Erythrophleum suaveolens (Guill. \& Perr.) Brenan et Stemonocoleus micranthus Harms. Malgré la prévalence de cette pathologie au Cameroun, très peu de travaux se sont consacrés à l'étude des plantes utilisées traditionnellement et en premier recours par les populations de l'arrondissement d'Akonolinga, l'un des principaux centres d'endémisme de cette pathologie. Afin d'explorer et d'accroître le potentiel en plantes médicinales traditionnellement utilisées contre cette pathologie, la présente étude s'est proposée d'identifier les plantes médicinales utilisées dans le traitement de l'UB dans l'arrondissement d'Akonolinga et de caractériser les recettes élaborées à base de ces plantes.

\section{MATÉRIEL ET MÉTHODES}

\section{Site d'étude}

L'étude s'est déroulée dans la localité d'Akonolinga et ses environs. Akonolinga est le chef-lieu du département du Nyong et Mfoumou, situé à $130 \mathrm{~km}$ de Yaoundé, entre $3^{\circ} 31^{\prime}-4^{\circ} 20^{\prime}$ latitude Nord et $11^{\circ} 55^{\prime}-12^{\circ} 30^{\prime}$ longitude Est et couvre une superficie d'environ $4209 \quad \mathrm{~km}^{2}$ (Figure 1). L'arrondissement d'Akonolinga couvre une superficie d'environ $4209 \mathrm{~km}^{2}$.

\section{Collecte des données}

Des enquêtes préliminaires ont permis de consulter la liste des tradipraticiens opérant dans le domaine de l'UB à Akonolinga. Les informations portant sur l'identité et la 
localisation de ces derniers ont été recueillies auprès des personnes ressources, notamment les anciens malades, les patriarches autochtones, les proches parents d'anciens malades; et également le personnel de santé de l'hôpital de district, en particulier les infirmiers ayant fait partie de l'équipe des Médecins Sans Frontières (MSF) lors des campagnes de prise en charge des patients atteints d'UB. La fiche d'enquête, élaborée selon le canevas proposé par Adjanohoun et al. (1994) pour la banque de données de Médecine Traditionnelle et Pharmacopée (PHARMEL) - Notice pour la collecte et l'entrée des données, a été administrée auprès de ceux des tradithérapeutes qui ont accepté de participer à cette étude et de communiquer leurs connaissances et savoirs-faire thérapeutiques. L'entretien était mené en langue locale et l'observation participative des séances de préparation des remèdes et des soins administrés à base de ces remèdes aux patients ont permis d'enregistrer les informations relatives aux organes d'espèces de plantes récoltées, ainsi qu'au mode de préparation et d'administration des remèdes.

\section{Récolte et identification de spécimens d'herbier \\ Les plantes indiquées par les} tradipraticiens ont été systématiquement photographiées et des échantillons botaniques ont été récoltés et ensuite déterminés au laboratoire de Systématique et Ethnobotanique de la Faculté des Sciences de l'Université de Yaoundé 1. Les vérifications de ces déterminations ont été faites par comparaison avec des spécimens de l'Herbier National du Cameroun à Yaoundé, avec la précieuse collaboration de Dr JM Onana, alors Chef de cette institution.

\section{Analyse statistique}

Les données obtenues ont été codifiées suivant le canevas proposé par Adjanohoun et al. (1994), puis enregistrées dans le tableur Excel de Microsoft 2013. Les graphiques ont été réalisés à partir des tableaux croisés dynamiques. Les espèces citées dans les différentes recettes ont été caractérisées par leur type morphologique tel que défini par Adjanohoun et al. (1994); Kidik Pouka et al. (2015). L'analyse, d'après Tardio et Pardo-deSantayana (2008), Andrade-Cetto et Heinrich (2011), Houndje et al. (2016), des citations d'usages basées sur le calcul de la fréquence relative de citations pour un usage donné, a permis d'établir des indicateurs sur les valeurs d'usage attribuées aux plantes recensées. La fréquence de citation $(\mathrm{Fc})$ des plantes par a été calculée selon la formule :

$$
\mathrm{Fc}=\frac{\mathrm{NCPC}}{\mathrm{NTCP}} \times 100
$$

avec $\mathrm{NCPC}=$ Nombre de citations pour la plante considérée $; \mathrm{NTCP}=$ Nombre total de citations pour toutes les plantes.

L'importance des espèces a été évaluée par leur poids de citation $\mathrm{Fc}$, le nombre de similitudes d'emplois relevées entre différents informateurs, l'indice d'intérêt thérapeutique et la convergence d'usage. Une plante ou une recette fait l'objet d'une similitude d'emploi lorsqu'elle est utilisée par au moins deux différents informateurs interrogés dans l'arrondissement d'Akonolinga. L'indice d'intérêt thérapeutique (IT) a été évalué sur la base de leur simple présence ou absence dans les différentes recettes indiquées par les informateurs. Lorsqu'une plante est utilisée dans au moins deux différentes régions géographiquement distinctes, elle fait l'objet d'une convergence d'usage. 


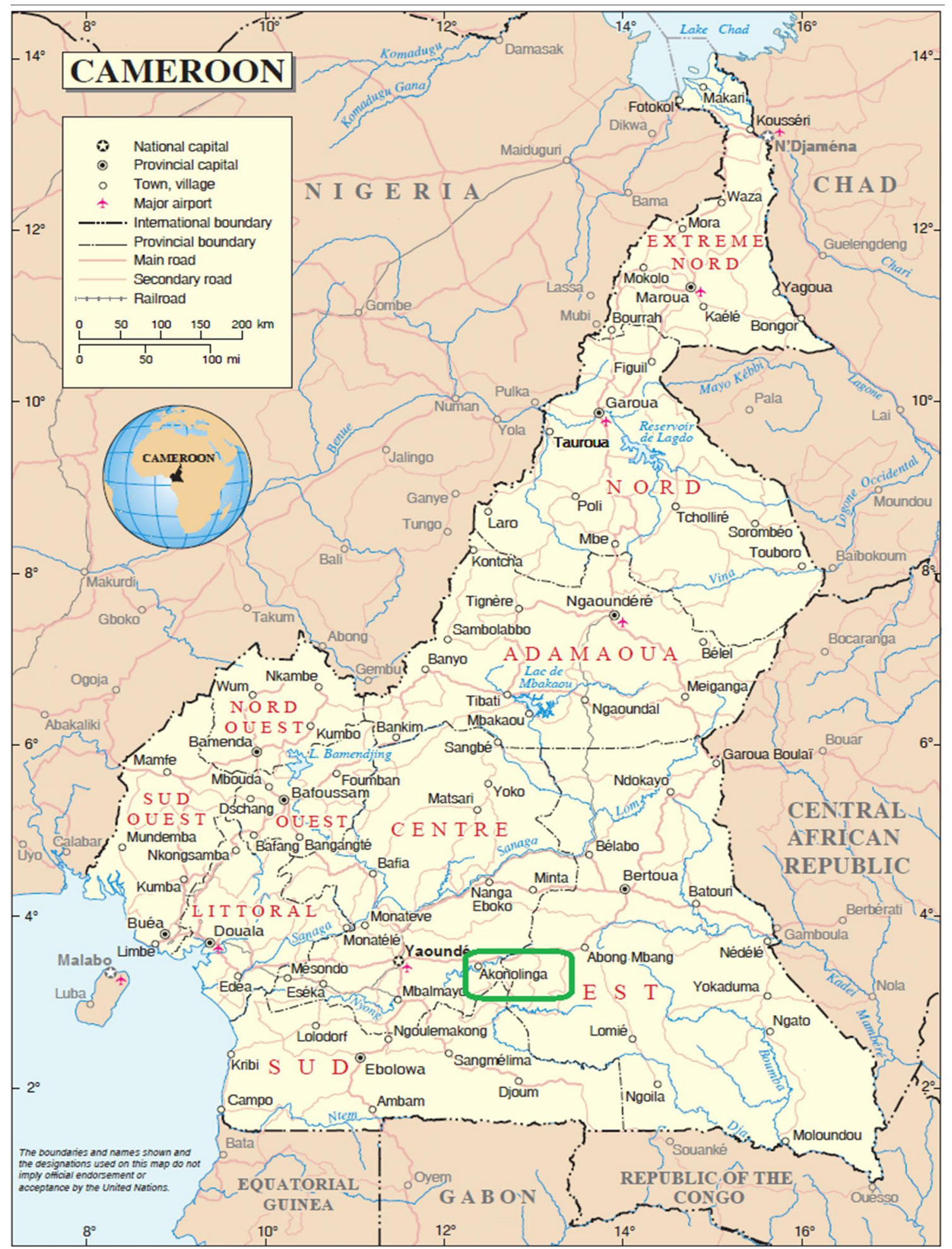

Figure 1: Carte du Cameroun montrant la région d'étude dans l'arrondissement d'Akonolinga (Source : Nations Unies, Carte ํo 4227 Rév. 2, 2015). 


\section{RESULTATS}

Caractéristiques ethnobotaniques des plantes utilisées pour le traitement de l'UB

Des vingt tradithérapeutes indiqués par les personnes ressources consultées, seulement neuf tradipraticiens, dont trois femmes (soit 33,33\%) et six hommes avec un âge variant entre 40 et 90 ans, repartis dans les huit villages, ont accepté de partager leur savoir-faire, en nous permettant d'assister à des séances de traitement et en nous décrivant les espèces utilisées, le principe, les moyens et techniques de traitement. De ces neuf tradithérapeutes, un total de 26 recettes, faisant intervenir 25 espèces médicinales appartenant à 25 genres et 20 familles a été recensé. La liste totale des espèces indiquées dans le traitement de l'ulcère de Buruli à Akonolinga et ses environs est présentée dans le Tableau 1; avec leur famille botanique, le nom vernaculaire et le numéro de récolte du spécimen d'herbier, le type morphologique de la plante, l'organe de plante utilisé ou la drogue, le mode de préparation des recettes, ainsi que le mode d'administration des remèdes. Pour chaque espèce inventoriée, les numéros des recettes dans lesquelles cette espèce est un constituant, de même que les numéros des tradithérapeutes ou informateurs ayant indiqué ces recettes, et le nombre total de citations d'usage sont également reportés dans ce tableau.

Les familles botaniques les plus représentées en nombre d'espèces sont les Rubiaceae et Mimosaceae avec chacune 3 espèces caractéristiques; suivies des Combretaceae et Cucurbitaceae avec chacune 2 espèces. Les autres familles ne comptent qu'une seule espèce représentative. Les espèces végétales les plus citées sont : Musa paradisiaca $(28,4 \%$ de citations), Petersianthus macrocarpus $(15,9 \%)$, Terminalia superba $(6,8 \%)$, Cassia spectabilis (6,8\%). Ceiba pentandra (4,5\%), Harungana madagascariensis $(4,5 \%)$ et Momordica cabraei (4,5\%). Parmi les 25 espèces recensées dans l'arrondissement d'Akonolinga et servant à traiter traditionnellement l'UB, neuf espèces apparaissent importantes car citées par au moins deux informateurs (Tableau 1). Ce sont notamment: Musa paradisiaca (9 informateurs), Petersianthus macrocarpus (6), Momordica cabraei (4), Cassia spectabilis (3), Citrus medica (3), Mitracarpus villosus (3), Terminalia superba (3), Ceiba pentandra (2) et Ipomoea aquatica (2).
De toutes les 26 recettes recensées, une seule recette $(3,85 \%)$ a fait appel à 4 différentes espèces de plantes, six autres $(23,08 \%)$ à 3 différentes espèces, 13 recettes $(50 \%)$ ont fait intervenir 2 différentes espèces et 6 recettes $(23,08 \%)$ ont fait appel à une seule espèce végétale (Tableau 2). L'évaluation de l'intérêt thérapeutique (IT), basée sur la simple présence ou absence des espèces dans les recettes, permet de retenir six espèces intéressantes, car intervenant dans au moins deux recettes et ayant un indice IT > 0,04. Ce sont notamment: Musa paradisiaca (0,69), Petersianthus macrocarpus $(0,27)$, Terminalia superba $(0,15)$, Cassia spectabilis, Ceiba pentandra et Mitracarpus villosus $(0,08$ chacune). Parmi les 25 espèces recensées, les herbacées $(48,2 \%)$ et les arbres $(41,1 \%)$ ont été les types morphologiques les plus utilisés, comparativement aux arbustes et lianes ligneuses $(5,4 \%$ chacun $)$.

\section{Caractéristiques des recettes utilisées dans le traitement de l'ulcère de Buruli}

Toutes les 26 recettes étaient constituées essentiellement d'espèces végétales (Tableau 1), cependant, certaines contenaient de la poudre de coquille de tortue (Recette numéro 7 ou R7), de la poudre d'os d'hiboux (R2, R3, R9, R23) ou encore du sel gemme (R23). Pour la préparation de ces recettes, l'écorce de tige $(41,1 \%)$, les tiges $(26,8 \%)$ et les feuilles (25\%) étaient les organes ou drogues les plus utilisés, en nombre de citations, comparativement aux autres parties de plantes (Figure 2). La décoction $(64,3 \%)$ et le pilage $(23,2 \%)$ constituaient les deux modes de préparation les plus fréquents en nombre de citations (Figure 3) et également en proportion d'espèces. Le mode d'administration ou d'emploi des remèdes ainsi obtenus était essentiellement par voie externe et s'opérait : soit par massage et désinfection de la lésion avec les décoctions préparées $(64,3 \%)$, soit par application locale en cataplasme $(14,3 \%)$ ou par badigeonnage $(3,6 \%)$, soit par projection sur les lésions ou plaies ulcéreuses, de la préparation contenue dans des fragments de feuilles de bananier préalablement ramollies au feu de bois (14,3\%). L'épandage ou saupoudrage et la friction des lésions étaient les modes les moins employés (Figure 4). 
H. N. BAYAGA et al. / Int. J. Biol. Chem. Sci. 11(4): 1523-1541, 2017

Tableau 1 : Informations relatives aux plantes et aux recettes utilisées dans le traitement traditionnel de l’Ulcère de Buruli selon 9 tradithérapeutes à Akonolinga.

\begin{tabular}{|c|c|c|c|c|c|c|c|c|c|}
\hline Espèces & $\begin{array}{l}\text { Familles } \\
\text { botaniques }\end{array}$ & $\begin{array}{l}\text { Nom V. } \\
\left(N^{\circ} \mathrm{SH}\right)\end{array}$ & $\begin{array}{l}\text { Type } \\
\text { morpho- } \\
\text { logique }\end{array}$ & Organe & $\begin{array}{l}\text { Mode de } \\
\text { préparation }\end{array}$ & $\begin{array}{l}\text { Mode d'admi- } \\
\text { nistration }\end{array}$ & $\mathbf{N}^{\circ}$ Recette & $\begin{array}{l}\mathrm{N}^{\circ} \text { Infor- } \\
\text { mateur }\end{array}$ & $\mathbf{C}$ \\
\hline $\begin{array}{l}\text { Aframomum melegueta } \\
\text { K. Schum. }\end{array}$ & Zingiberaceae & $\begin{array}{l}\text { Ndong } \\
\text { (BHN1) }\end{array}$ & Herbe & graine & Pilage & Projection & R14 & 1 & 1 \\
\hline $\begin{array}{l}\text { Albizia gummifera } \\
\text { (J.F. Gmel.) C.A. Sm. }\end{array}$ & Fabaceae & $\begin{array}{l}\text { Isack } \\
\text { (BHN2) }\end{array}$ & Arbre & $\begin{array}{l}\text { écorce de } \\
\text { tige }\end{array}$ & Décoction & $\begin{array}{l}\text { Massage et } \\
\text { désinfection de la } \\
\text { lésion }\end{array}$ & R25 & 3 & 1 \\
\hline Allium sativum Linn. & Amarylli-daceae & $\begin{array}{l}\text { Ail } \\
(\mathrm{BHN} 4) \\
\end{array}$ & Herbe & fruit & Pilage & $\begin{array}{l}\text { Application locale par } \\
\text { badigeonnage }\end{array}$ & R7 & 8 & 1 \\
\hline Cassia spectabilis DC. & Fabaceae & $\begin{array}{l}\text { Assilkông } \\
\text { (BHN5) }\end{array}$ & Arbre & $\begin{array}{l}\text { écorce de } \\
\text { tige }\end{array}$ & Décoction & $\begin{array}{l}\text { Massage et } \\
\text { désinfection de la } \\
\text { lésion }\end{array}$ & $\mathrm{R} 1 ; \mathrm{R} 8$ & $2 ; 7 ; 3$ & 6 \\
\hline $\begin{array}{l}\text { Ceiba pentandra (L.) } \\
\text { Gaerth. }\end{array}$ & Bombacaceae & $\begin{array}{l}\text { Douma } \\
\text { (BHN6) }\end{array}$ & Arbre & $\begin{array}{l}\text { écorce de } \\
\text { tige }\end{array}$ & Décoction & $\begin{array}{l}\text { Massage et } \\
\text { désinfection de la } \\
\text { lésion }\end{array}$ & R19; R22 & $4 ; 5$ & 4 \\
\hline Citrus medica L. & Rutaceae & $\begin{array}{l}\text { Osanga } \\
\text { (BHN7) }\end{array}$ & Arbuste & fruit & Expression & Friction & R6 & $2 ; 3 ; 8$ & 3 \\
\hline $\begin{array}{l}\text { Cogniauxia podolaena } \\
\text { Baill }\end{array}$ & Cucurbitaceae & $\begin{array}{l}\text { Mbomvae } \\
\text { (BHN8) }\end{array}$ & Liane & feuilles & Pilage & $\begin{array}{l}\text { Application locale en } \\
\text { cataplasme }\end{array}$ & R12 & 6 & 1 \\
\hline $\begin{array}{l}\text { Combretum zenkeri } \\
\text { Engl. \& Diels. }\end{array}$ & Combretaceae & $\begin{array}{l}\text { Otugudu } \\
\text { (BHN9) }\end{array}$ & Herbe & feuilles & Pilage & Projection & R3 & 7 & 1 \\
\hline Costus afer Ker-Gawl & Costaceae & $\begin{array}{l}\text { Myan } \\
(\mathrm{BHN} 10)\end{array}$ & Herbe & tige & Pilage & Projection & R14 & 1 & 1 \\
\hline
\end{tabular}


H. N. BAYAGA et al. / Int. J. Biol. Chem. Sci. 11(4): 1523-1541, 2017

\begin{tabular}{|c|c|c|c|c|c|c|c|c|c|}
\hline Elaeis guineensis Jacq. & Araceae & $\begin{array}{l}\text { Alen } \\
(\mathrm{BHN} 11)\end{array}$ & Arbre & amande & Pilage & $\begin{array}{l}\text { Application locale par } \\
\text { badigeonnage }\end{array}$ & R7 & 8 & 1 \\
\hline \multirow[t]{2}{*}{$\begin{array}{l}\text { Erythrococca africana } \\
\text { Baill. }\end{array}$} & Euphorbiaceae & $\begin{array}{l}\text { Efulkumba } \\
\text { BHN12) }\end{array}$ & Herbe & feuilles & Pilage & Projection & $\mathrm{R} 2$ & 7 & 1 \\
\hline & & & & feuilles & Trituration & $\begin{array}{l}\text { Application locale en } \\
\text { cataplasme }\end{array}$ & $\mathrm{R} 2$ & 7 & 1 \\
\hline $\begin{array}{l}\text { Harungana } \\
\text { madagascariensis } \\
\text { Lam.exp Poir }\end{array}$ & Hypericaceae & $\begin{array}{l}\text { Atondog } \\
(\mathrm{BHN} 14)\end{array}$ & Arbre & $\begin{array}{l}\text { écorce de } \\
\text { tige }\end{array}$ & Décoction & $\begin{array}{l}\text { Massage et } \\
\text { désinfection de la } \\
\text { lésion }\end{array}$ & R1 & 7 & 4 \\
\hline $\begin{array}{l}\text { Hymenocardia lyrata } \\
\text { Tul. }\end{array}$ & Phyllanthaceae & $\begin{array}{l}\text { Isang } \\
(\mathrm{BHN} 15)\end{array}$ & Arbuste & feuilles & Pilage & Projection & R23 & 5 & 2 \\
\hline $\begin{array}{l}\text { Ipomoea aquatica } \\
\text { Forssk. }\end{array}$ & $\begin{array}{l}\text { Convolvula- } \\
\text { ceae }\end{array}$ & $\begin{array}{l}\text { Atêt nko'o } \\
\text { (BHN16) }\end{array}$ & Liane & feuilles & Pilage & Projection & R9 & $2 ; 3$ & 2 \\
\hline $\begin{array}{l}\text { Mammea africana } \\
\text { Sabine }\end{array}$ & Clusiaceae & $\begin{array}{l}\text { Abôt/Abod } \\
\text { zok } \\
\text { (BHN17) }\end{array}$ & Arbre & $\begin{array}{l}\text { écorce de } \\
\text { tige }\end{array}$ & Décoction & $\begin{array}{l}\text { Massage et } \\
\text { désinfection de la } \\
\text { lésion }\end{array}$ & R10 & 6 & 1 \\
\hline \multirow[t]{2}{*}{$\begin{array}{l}\text { Mitracarpus villosus } \\
\text { (SW) DC.S }\end{array}$} & Rubiaceae & $\begin{array}{l}\text { Oyem ze } \\
(\text { BHN18) }\end{array}$ & Herbe & feuilles & Pilage & $\begin{array}{l}\text { Application locale en } \\
\text { cataplasme }\end{array}$ & $\mathrm{R} 4$ & $2 ; 9$ & 2 \\
\hline & & & & & & Projection & $\mathrm{R} 3$ & 7 & 1 \\
\hline $\begin{array}{l}\text { Momordica cabraei } \\
\text { (Cogn) Jeffrey }\end{array}$ & Combretaceae & $\begin{array}{l}\text { Ekok } \\
\text { (BHN19) }\end{array}$ & Liane & feuilles & Pilage & $\begin{array}{l}\text { Application locale en } \\
\text { cataplasme }\end{array}$ & R24 & $2 ; 3 ; 4 ; 5$ & 4 \\
\hline
\end{tabular}


H. N. BAYAGA et al. / Int. J. Biol. Chem. Sci. 11(4): 1523-1541, 2017

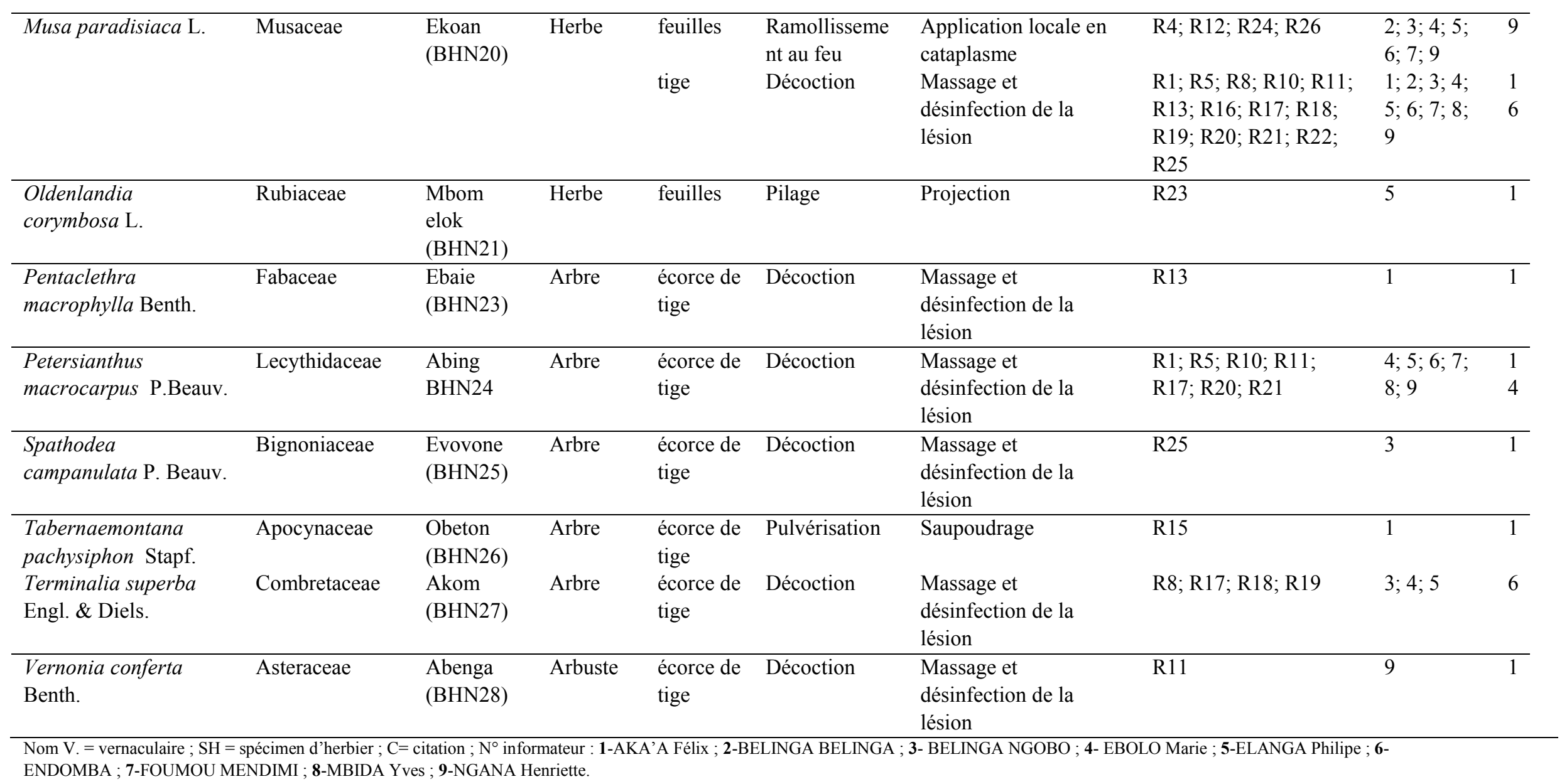


H. N. BAYAGA et al. / Int. J. Biol. Chem. Sci. 11(4): 1523-1541, 2017

Tableau 2 : Espèces végétales d'intérêt pour leur usage en traitement traditionnel de l'Ulcère de Buruli à Akonolinga.

\begin{tabular}{|c|c|c|c|c|c|c|c|c|c|c|c|c|c|c|c|c|c|c|c|c|c|c|c|c|c|c|c|c|c|}
\hline Espèces & $\mathbf{C}$ & $\bar{\simeq}$ & $\stackrel{\sim}{\simeq}$ & $\widetilde{n}$ & $\underset{\simeq}{\check{q}}$ & $\stackrel{n}{\simeq}$ & $\ddot{q}$ & 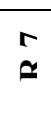 & $\begin{array}{l}\infty \\
\simeq\end{array}$ & $\hat{a}$ & $\stackrel{0}{\simeq}$ & $\approx$ & $\frac{1}{\simeq}$ & $\stackrel{m}{\simeq}$ & \pm & $\frac{n}{\simeq}$ & $\frac{0}{2}$ & $\frac{r}{2}$ & $\stackrel{\infty}{\simeq}$ & $\frac{a}{\simeq}$ & $\underset{\sim}{\simeq}$ & $\tilde{\sim}$ & $\underset{\simeq}{\simeq}$ & $\stackrel{\widetilde{N}}{\simeq}$ & $\stackrel{\mathbb{N}}{\simeq}$ & $\stackrel{\mathscr{N}}{\simeq}$ & $\stackrel{0}{\sim}$ & 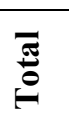 & IT \\
\hline $\begin{array}{l}\text { Aframomum } \\
\text { melegueta }\end{array}$ & 1 & 0 & 0 & 0 & 0 & 0 & 0 & 0 & 0 & 0 & 0 & 0 & 0 & 0 & 1 & 0 & 0 & 0 & 0 & 0 & 0 & 0 & 0 & 0 & 0 & 0 & 0 & 1 & 0,04 \\
\hline Albizia gummifera & 1 & 0 & 0 & 0 & 0 & 0 & 0 & 0 & 0 & 0 & 0 & 0 & 0 & 0 & 0 & 0 & 0 & 0 & 0 & 0 & 0 & 0 & 0 & 0 & 0 & 1 & 0 & 1 & 0,04 \\
\hline Allium sativum & 1 & 0 & 0 & 0 & 0 & 0 & 0 & 1 & 0 & 0 & 0 & 0 & 0 & 0 & 0 & 0 & 0 & 0 & 0 & 0 & 0 & 0 & 0 & 0 & 0 & 0 & 0 & 1 & 0,04 \\
\hline Cassia spectabilis & 6 & 1 & 0 & 0 & 0 & 0 & 0 & 0 & 1 & 0 & 0 & 0 & 0 & 0 & 0 & 0 & 0 & 0 & 0 & 0 & 0 & 0 & 0 & 0 & 0 & 0 & 0 & 2 & 0,08 \\
\hline Ceiba pentandra & 4 & 0 & 0 & 0 & 0 & 0 & 0 & 0 & 0 & 0 & 0 & 0 & 0 & 0 & 0 & 0 & 0 & 0 & 0 & 1 & 0 & 0 & 1 & 0 & 0 & 0 & 0 & 2 & 0,08 \\
\hline Citrus medica & 3 & 0 & 0 & 0 & 0 & 0 & 1 & 0 & 0 & 0 & 0 & 0 & 0 & 0 & 0 & 0 & 0 & 0 & 0 & 0 & 0 & 0 & 0 & 0 & 0 & 0 & 0 & 1 & 0,04 \\
\hline $\begin{array}{l}\text { Cogniauxia } \\
\text { podolaena }\end{array}$ & 1 & 0 & 0 & 0 & 0 & 0 & 0 & 0 & 0 & 0 & 0 & 0 & 1 & 0 & 0 & 0 & 0 & 0 & 0 & 0 & 0 & 0 & 0 & 0 & 0 & 0 & 0 & 1 & 0,04 \\
\hline $\begin{array}{l}\text { Combretum } \\
\text { zenkeri }\end{array}$ & 1 & 0 & 0 & 1 & 0 & 0 & 0 & 0 & 0 & 0 & 0 & 0 & 0 & 0 & 0 & 0 & 0 & 0 & 0 & 0 & 0 & 0 & 0 & 0 & 0 & 0 & 0 & 1 & 0,04 \\
\hline Costus afer & 1 & 0 & 0 & 0 & 0 & 0 & 0 & 0 & 0 & 0 & 0 & 0 & 0 & 0 & 1 & 0 & 0 & 0 & 0 & 0 & 0 & 0 & 0 & 0 & 0 & 0 & 0 & 1 & 0,04 \\
\hline Elaeis guineensis & 1 & 0 & 0 & 0 & 0 & 0 & 0 & 1 & 0 & 0 & 0 & 0 & 0 & 0 & 0 & 0 & 0 & 0 & 0 & 0 & 0 & 0 & 0 & 0 & 0 & 0 & 0 & 1 & 0,04 \\
\hline $\begin{array}{l}\text { Erythrococca } \\
\text { africana }\end{array}$ & 2 & 0 & 1 & 0 & 0 & 0 & 0 & 0 & 0 & 0 & 0 & 0 & 0 & 0 & 0 & 0 & 0 & 0 & 0 & 0 & 0 & 0 & 0 & 0 & 0 & 0 & 0 & 1 & 0,04 \\
\hline $\begin{array}{l}\text { Harungana } \\
\text { madagascariensis }\end{array}$ & 4 & 1 & 0 & 0 & 0 & 0 & 0 & 0 & 0 & 0 & 0 & 0 & 0 & 0 & 0 & 0 & 0 & 0 & 0 & 0 & 0 & 0 & 0 & 0 & 0 & 0 & 0 & 1 & 0,04 \\
\hline $\begin{array}{l}\text { Hymenocardia } \\
\text { lyrata }\end{array}$ & 2 & 0 & 0 & 0 & 0 & 0 & 0 & 0 & 0 & 0 & 0 & 0 & 0 & 0 & 0 & 0 & 0 & 0 & 0 & 0 & 0 & 0 & 0 & 1 & 0 & 0 & 0 & 1 & 0,04 \\
\hline
\end{tabular}


H. N. BAYAGA et al. / Int. J. Biol. Chem. Sci. 11(4): 1523-1541, 2017

\begin{tabular}{|c|c|c|c|c|c|c|c|c|c|c|c|c|c|c|c|c|c|c|c|c|c|c|c|c|c|c|c|c|c|}
\hline Ipomoea aquatica & 2 & 0 & 0 & 0 & 0 & 0 & 0 & 0 & 0 & 1 & 0 & 0 & 0 & 0 & 0 & 0 & 0 & 0 & 0 & 0 & 0 & 0 & 0 & 0 & 0 & 0 & 0 & 1 & 0,04 \\
\hline Mammea africana & 1 & 0 & 0 & 0 & 0 & 0 & 0 & 0 & 0 & 0 & 1 & 0 & 0 & 0 & 0 & 0 & 0 & 0 & 0 & 0 & 0 & 0 & 0 & 0 & 0 & 0 & 0 & 1 & 0,04 \\
\hline $\begin{array}{l}\text { Mitracarpus } \\
\text { villosus }\end{array}$ & 3 & 0 & 0 & 1 & 1 & 0 & 0 & 0 & 0 & 0 & 0 & 0 & 0 & 0 & 0 & 0 & 0 & 0 & 0 & 0 & 0 & 0 & 0 & 0 & 0 & 0 & 0 & 2 & $\mathbf{0 , 0 8}$ \\
\hline $\begin{array}{l}\text { Momordica } \\
\text { cabraei }\end{array}$ & 4 & 0 & 0 & 0 & 0 & 0 & 0 & 0 & 0 & 0 & 0 & 0 & 0 & 0 & 0 & 0 & 0 & 0 & 0 & 0 & 0 & 0 & 0 & 0 & 1 & 0 & 0 & 1 & 0,04 \\
\hline Musa paradisiaca & 25 & 1 & 0 & 0 & 1 & 1 & 0 & 0 & 1 & 0 & 1 & 1 & 1 & 1 & 0 & 0 & 1 & 1 & 1 & 1 & 1 & 1 & 1 & 0 & 1 & 1 & 1 & 18 & 0,69 \\
\hline $\begin{array}{l}\text { Oldenlandia } \\
\text { corymbosa }\end{array}$ & 1 & 0 & 0 & 0 & 0 & 0 & 0 & 0 & 0 & 0 & 0 & 0 & 0 & 0 & 0 & 0 & 0 & 0 & 0 & 0 & 0 & 0 & 0 & 1 & 0 & 0 & 0 & 1 & 0,04 \\
\hline $\begin{array}{l}\text { Pentaclethra } \\
\text { macrophylla }\end{array}$ & 1 & 0 & 0 & 0 & 0 & 0 & 0 & 0 & 0 & 0 & 0 & 0 & 0 & 1 & 0 & 0 & 0 & 0 & 0 & 0 & 0 & 0 & 0 & 0 & 0 & 0 & 0 & 1 & 0,04 \\
\hline $\begin{array}{l}\text { Petersianthus } \\
\text { macrocarpus }\end{array}$ & 14 & 1 & 0 & 0 & 0 & 1 & 0 & 0 & 0 & 0 & 1 & 1 & 0 & 0 & 0 & 0 & 0 & 1 & 0 & 0 & 1 & 1 & 0 & 0 & 0 & 0 & 0 & 7 & $\mathbf{0 , 2 7}$ \\
\hline $\begin{array}{l}\text { Spathodea } \\
\text { campanulata }\end{array}$ & 1 & 0 & 0 & 0 & 0 & 0 & 0 & 0 & 0 & 0 & 0 & 0 & 0 & 0 & 0 & 0 & 0 & 0 & 0 & 0 & 0 & 0 & 0 & 0 & 0 & 1 & 0 & 1 & 0,04 \\
\hline $\begin{array}{l}\text { Tabernaemontana } \\
\text { pachysiphon }\end{array}$ & 1 & 0 & 0 & 0 & 0 & 0 & 0 & 0 & 0 & 0 & 0 & 0 & 0 & 0 & 0 & 1 & 0 & 0 & 0 & 0 & 0 & 0 & 0 & 0 & 0 & 0 & 0 & 1 & 0,04 \\
\hline $\begin{array}{l}\text { Terminalia } \\
\text { superba }\end{array}$ & 6 & 0 & 0 & 0 & 0 & 0 & 0 & 0 & 1 & 0 & 0 & 0 & 0 & 0 & 0 & 0 & 0 & 1 & 1 & 1 & 0 & 0 & 0 & 0 & 0 & 0 & 0 & 4 & 0,15 \\
\hline Vernonia conferta & 1 & 0 & 0 & 0 & 0 & 0 & 0 & 0 & 0 & 0 & 0 & 1 & 0 & 0 & 0 & 0 & 0 & 0 & 0 & 0 & 0 & 0 & 0 & 0 & 0 & 0 & 0 & 1 & 0,04 \\
\hline Total & 88 & 4 & 1 & 2 & 2 & 2 & 1 & 2 & 3 & 1 & 3 & 3 & 2 & 2 & 2 & 1 & 1 & 3 & 2 & 3 & 2 & 2 & 2 & 2 & 2 & 3 & 1 & 54 & 2,08 \\
\hline
\end{tabular}

Intérêt thérapeutique (IT) = présence (1) /absence (0) dans les recettes qui ne partagent pas les mêmes plantes ; $\mathrm{C}=$ citation en fréquence absolue ; $\mathrm{R}=$ recette. 


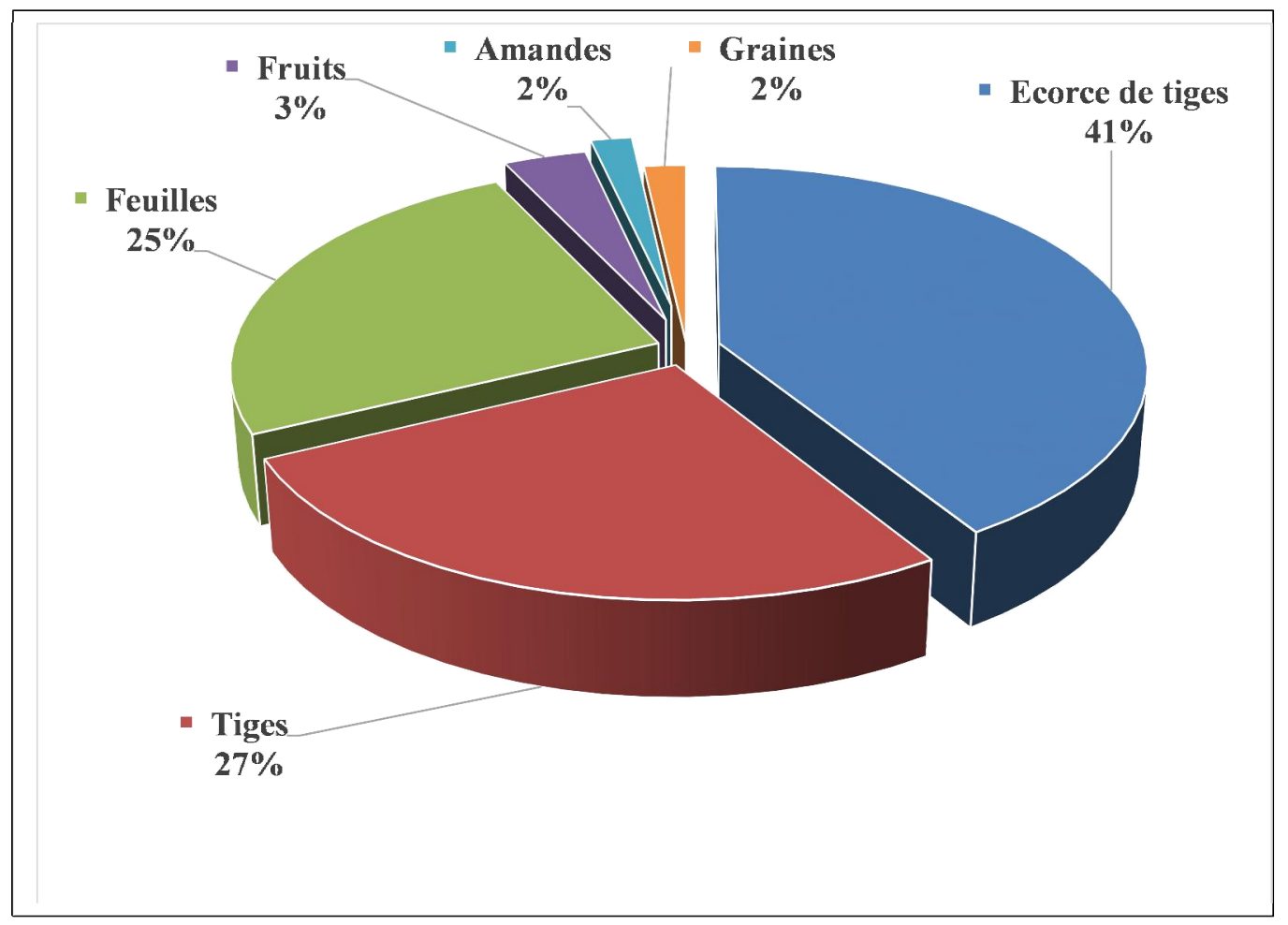

Figure 2 : Proportion (\% de citation) des parties d'organe de plantes utilisées dans la composition des recettes de traitement de l'Ulcère de Buruli.

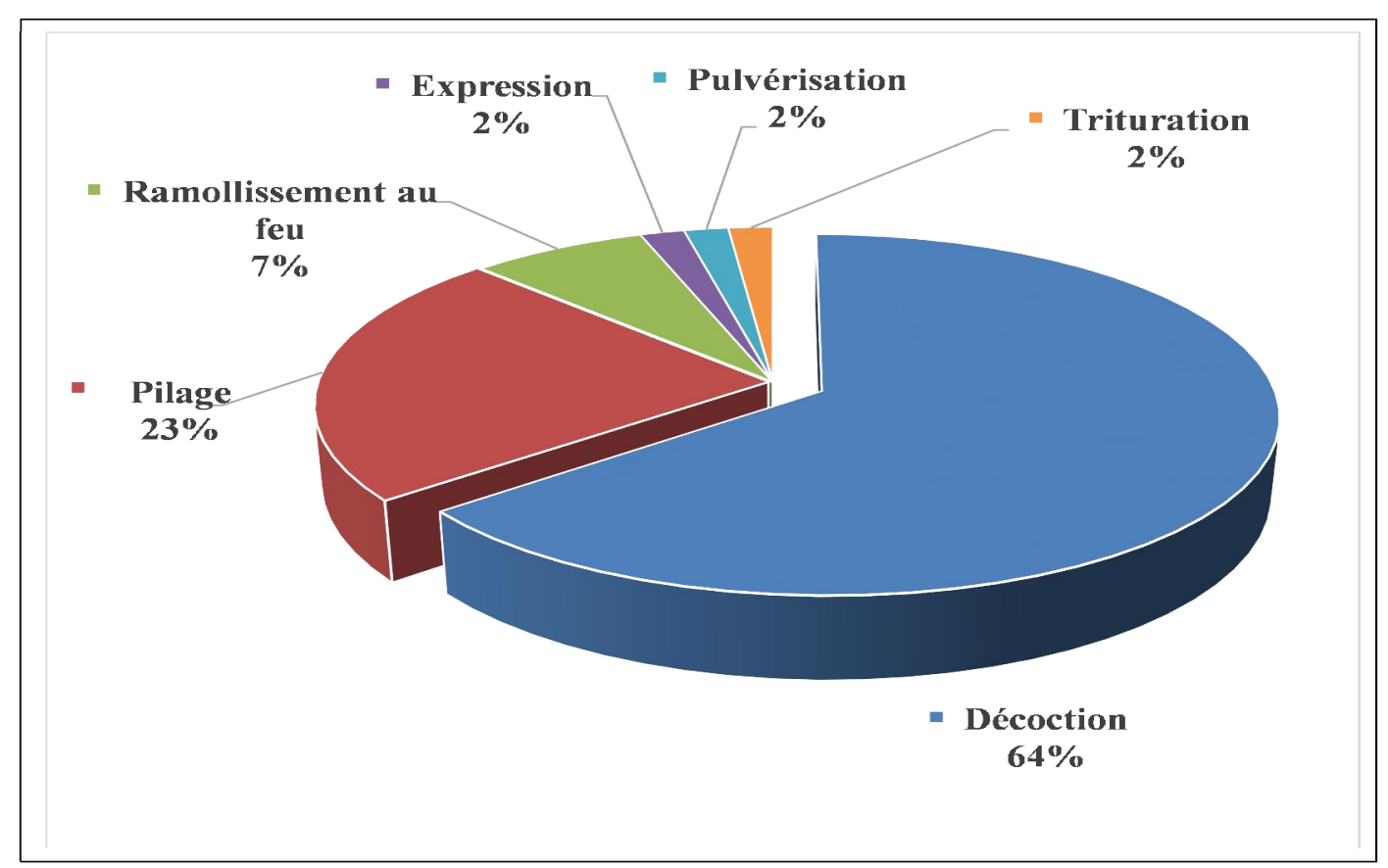

Figure 3 : Méthodes de préparation des recettes utilisées pour le traitement de l'Ulcère de Buruli à Akonolinga (\% de citation). 


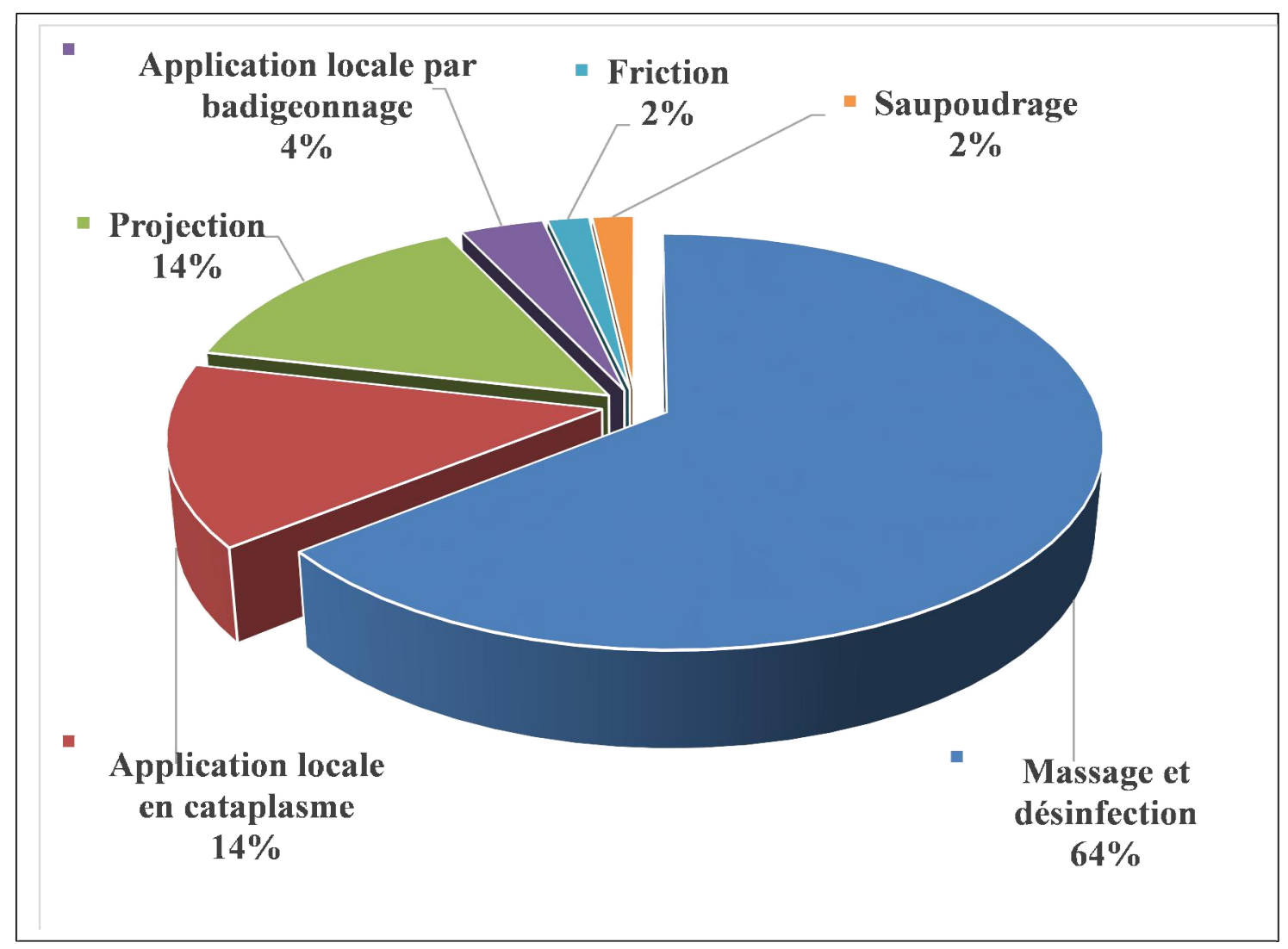

Figure 4 : Modalités d'administration des médicaments contre l’Ulcère de Buruli (\% de citation).

\section{DISCUSSION}

\section{Importance relative des espèces végétales}

L'étude du discours et des remèdes de la médecine traditionnelle, c'est-à-dire de la pharmacopée traditionnelle, se fait sur des critères ethnopharmacologiques, aux premiers rangs desquels l'analyse des citations d'usages, la recherche des similitudes d'emplois et la convergence d'usage (Tardio et Pardo-de-Santayana, 2008; Fleurentin et al., 2011). Parmi les 25 espèces recensées dans la région d'Akonolinga et servant à traiter traditionnellement l'UB, les neuf espèces importantes parce que citées par au moins deux informateurs sont: $M$. paradisiaca, $P$. macrocarpus, M. cabraei, C. spectabilis, $C$. medica, $M$. villosus, $T$. superba, $C$. pentandra et $I$. aquatica. Parmi les seize autres espèces, indiquées par un seul informateur, trois plantes sont indiquées dans le traitement traditionnel de l'UB en Afrique de l'Ouest, en l'occurrence Aframomum melegueta au Benin et au Ghana (Yemoa et al., 2008, 2011; Seefeld et al., 2013); Elaeis guineensis en Côte d'Ivoire (Adjet et al., 2013; 2016); Spathodea campanulata au Benin, Côte d'Ivoire et Ghana (Adjet et al., 2013; Sam et al., 2013; Addo et al., 2007; Yemoa et al., 2008; 2011). Les treize autres espèces, non indiquées dans le littérature comme plantes anti-ulcère de Buruli sont plutôt indiquées pour d'autres indications thérapeutiques telles que le diabète, la jaunisse, la gale, les céphalées et fièvres, la tuberculose, les dermatoses, l'ascite, l'épilepsie, les éruptions cutanées, la blennorragie, les vers intestinaux, l'hypertension artérielle, la typhoïde (Dongmo et al., 2007; N'guessan et al., 2009; Mangambu et al., 2014; Mpondo et al., 2015). 
La plupart des travaux ethnobotaniques et ethnopharmacologiques ont basé essentiellement l'importance relative des plantes sur le nombre de similitudes d'emplois relevées entre les différents informateurs (Diafouka, 1997; Betti, 2001; Zahoor et al., 2017). Cependant, comme l'a souligné Betti (2001), une plante intéressante ne peut être utilisée que par un seul guérisseur qui garde jalousement le secret de peur de perdre le monopole qu'il détient sur cette espèce. Et donc, le seul fait qu'une plante soit citée uniquement par un seul informateur ne suffit pas pour décider de son importance thérapeutique. A cause du caractère secret prononcé de la médecine traditionnelle, il y a des risques de laisser de côté des plantes intéressantes si l'on se limite uniquement aux similitudes d'emplois observées entre les différents informateurs. C'est ainsi que l'évaluation de l'intérêt thérapeutique (IT) a permis de retenir six autres espèces intéressantes $(M$. paradisiaca, $P$. macrocarpus, T. superba, C. spectabilis, $C$. pentandra et $M$. villosus), et qui sont également déjà dénombrées parmi les neuf espèces citées par au moins deux informateurs.

L'analyse de convergence d'usage entre différentes régions géographiques a montré que, de ces six espèces à IT $>0,04$, Musa parasidiaca est citée parmi les plantes utilisées contre l'UB en Côte d'Ivoire (Adjet et al., 2013) et Mitracarpus villosus est citée pour le même usage au Ghana (OwusuSekyere, 2012). Pour Fleurentin et al. (2011), les notions de convergence d'usage et de critère d'utilisation constituent deux notions clés validant à la fois l'approche ethnopharmacologique et l'outil de recherche et de sélection des espèces et/ou des préparations les plus utilisées et les plus significatives. En effet, l'ethnopharmacologue moderne s'intéresse aux convergences d'usages perçues comme des marqueurs d'activité biologiques, car il est saisissant de constater l'existence, pour un certain nombre de plantes, d'usages très similaires dans des lieux géographiques très différents. Cela s'est avéré pour plusieurs espèces de plantes telles que le papayer, mais également pour des espèces de diffusion plus restreinte et aux usages plus ciblés (Fleurentin et al., 2011).

$\mathrm{Au}$ total donc, cinq espèces (en gras et surlignées de gris dans le Tableau 2), à savoir Musa parasidiaca, Mitracarpus villosus, Aframomum melegueta, Elaeis guineensis et Spathodea campanulata, font partie des espèces aux convergences d'usages remarquables entre différentes régions géographiques; tandis que les vingt autres espèces constituent la contribution de la présente étude à la liste des plantes africaines utilisées en médecine traditionnelle contre l'Ulcère de Buruli. Aux premiers rangs de cette vingtaine d'espèces, sept d'entre elles (en gras dans le Tableau 2), notamment, Petersianthus macrocarpus, Momordica cabraei, Cassia spectabilis, Citrus medica, Terminalia superba, Ceiba pentandra et Ipomoea aquatica constituent l'autre groupe d'espèces les plus significatives, car citées par au moins deux informateurs et/ou intervenant dans au moins deux différentes recettes.

\section{Analyse des caractéristiques des recettes}

L'analyse des recettes de plantes utilisées pour traiter l'UB a fait ressortir que l'écorce de tiges, les tiges et les feuilles dominaient parmi les 6 types d'organes végétaux cités dans l'arrondissement d'Akonolinga. L'importance des tiges ici est à relativiser car elle ne concerne qu'une seule espèce, Musa paradisiaca qui intervient dans 18 recettes et dont les fibres de la tige servent comme gaze à imprégner par les décoctions préparées pour masser et désinfecter les lésions. C'est d'ailleurs ce qui justifie la fréquence de citation élevée de cette espèce, liée à son association fréquente dans ces 18 recettes indiquées par tous les neuf informateurs. La prépondérance de l'écorce de tiges dans les recettes pour le traitement de l'UB à Akonolinga est un trait caractéristique, également relevé par Betti (2001), pour la médecine traditionnelle du Dja, et Dibong et al. (2015) pour les plantes médicinales antihémorroïdaires des marchés et villages du 
Centre et du Littoral Cameroun; alors que l'essentiel des études éthnobotaniques et ethnopharmacologiques au Cameroun ou en Afrique, relèvent plutôt la prépondérance des feuilles par rapport aux autres organes utilisés dans la pharmacopée traditionnelle (Djafouka, 1997; Dibong et al., 2011; 2015 ; Ghourri et al., 2013) et même dans le traitement de l'UB en Afrique de l'Ouest (Tsouh Fokou et al., 2015). L'importance des feuilles dans les recettes contre l'UB dans l'arrondissement d'Akonolinga peut être directement mis en relation avec le spectre pondéré des types morphologiques des espèces utilisées, qui a montré une nette dominance des herbacées et pour lesquelles l'organe le plus exploité est la feuille.

$\mathrm{La}$ décoction était la forme pharmaceutique la plus indiquée dans le traitement de l'UB à Akonolinga suivie du pilât. Les travaux de Tsouh Fokou et al. (2015) ont également montré que la décoction était la forme pharmaceutique la plus employée en Afrique de l'Ouest pour les remèdes contre l'UB. Plusieurs autres travaux sur les plantes utilisées en pharmacopée traditionnelle soulignent également la prépondérance de cette forme pharmaceutique (Betti, 2001 ; Tsobou et al., 2013; Dibong et al., 2015;), qui semble présenter l'avantage d'extraire le maximum de substances végétales solubles dans l'eau bouillante. Tous les remèdes recensés à Akonolinga étaient administrés par voie externe, essentiellement en massage et désinfection, application de cataplasme ou en projection sur les lésions ou plaies. Par contre, Yemoa et al. (2008), Tsouh Fokou et al. (2015) ont indiqué une administration des formes préparées par voie interne et/ou externe au Bénin, Ghana et Côte d'Ivoire. La spécificité des recettes recensées dans l'arrondissement d'Akonolinga réside, dans la constance chez les 9 tradithérapeutes, de l'usage des fibres de tige du bananierplantain, Musa parasidiaca, imprégnées des différents décoctés pour le massage et la désinfection des lésions ulcéreuses.

\section{Complexité des préparations}

La plupart des 26 recettes recensées à Akonolinga étaient constituées par au moins deux espèces végétales. Plusieurs travaux réalisés sur les traitements traditionnels en Afrique ont souligné cette complexité dans la préparation et le contenu des remèdes traditionnels, qui comptent rarement une seule espèce végétale, avec parfois des recettes contenant plus de quatre ingrédients végétaux différents. Cette forme d'associations de diverses espèces végétales dans les traitements ou recettes pourrait présenter des risques d'interaction ou de toxicité d'après Yemoa et al. (2008). Cependant, Fleurentin et al. (2011) soulignent que les activités thérapeutiques réelles de certaines plantes médicinales, ne peuvent être expliquées par la seule présence de l'un ou l'autre des constituants. En effet, dans ces mélanges complexes que sont les soupes moléculaires d'extraits de diverses plantes, c'est l'association ou la synergie qui existe entre les constituants, qui est très souvent responsable de l'effet recherché. Pour ces auteurs, quelle que soit la plante médicinale considérée, on peut affirmer sans se tromper que ses effets ne sont jamais dus à une seule molécule agissant sur une seule cible; il s'agit toujours d'un cocktail de molécules de structures chimiques très diverses agissant à plusieurs niveaux en combinaison, dont certaines indirectement actives ou facilitant l'activité, participent directement à l'effet observé.

Ces mélanges de molécules complexes, potentiellement synergiques, ont toujours été difficiles à travailler, mais depuis peu, l'approche ethnopharmacologique, se dotant de nouveaux paradigmes conceptuels et de nouveaux outils analytiques, peut maintenant à nouveau repenser l'activité des remèdes comme des ensembles complexes, interactifs. $\mathrm{Ce}$ raisonnement ethnopharmacologique, qui tient une place prépondérante dans la recherche de nouveaux traitements de demain, met donc de plus en plus l'accent sur les mélanges, les synergies, les pro-drogues, et toutes les molécules aux effets modulateurs d'activité, avec comme principaux défis de 
s'intéresser aux préparations complexes et à ce qu'elles peuvent donner comme nouvelles pistes pour concevoir des traitements pluricomposants ou plurithérapies, plus efficaces et moins iatrogènes comparativement aux médicaments actuels de synthèse.

\section{Conclusion}

L'exploration du potentiel en plantes médicinales traditionnellement utilisées contre l'ulcère de Buruli dans l'arrondissement d'Akonolinga a permis d'identifier cinq espèces faisant partie des espèces aux convergences d'usages remarquables entre différentes régions géographiques et sept autres espèces significatives, car citées par au moins deux informateurs et/ou intervenant dans au moins deux différentes recettes. En mettant en évidence ces douze (12) espèces végétales d'intérêt, la présente étude a une applicabilité directe intéressant la recherche en innovation thérapeutique, par le raisonnement ethnopharmacologique, en concourant ainsi à la sélection des espèces et à l'orientation des évaluations chimiques, pharmacologiques et/ou cliniques prioritaires conduisant à l'identification de nouvelles molécules et/ou au développement de nouveaux médicaments dont l'Afrique a besoin pour la prise en charge de ses pathologies endémiques.

\section{CONFLIT D'INTERETS} d'intérêts.

Les auteurs ne déclarent aucun conflit

\section{CONTRIBUTIONS DES AUTEURS}

HNB a contribué à la conception et à la structuration de l'étude, à la récolte des données sur le terrain et à la rédaction de la première mouture de l'article. NMG a contribué à la conception et à la structuration de l'étude, à la recherche documentaire, à l'analyse et à l'interprétation des données, à la rédaction, révision critique, discussion et finalisation de l'article. EHB a contribué à la conception et à la structuration de l'étude.

\section{REMERCIEMENTS}

Nous n'aurions certainement pas pu mener à bien cette étude sans la précieuse collaboration de nos informateurs, il s'agit de Mesdames Marie EBOLO, ENDOMBA et Henriette NGANA ; Messieurs Felix AKA'A, Philipe ELANGA, BELINGA BELINGA, BELINGA NGOOBO, FOUMOU MENDIMI, et Yves MBIDA. Ils ont accepté de nous accueillir et de partager gracieusement leurs savoirs-faire et les recettes de traitement à base de plantes contre l'Ulcère de Buruli. Nous leur témoignons notre profonde gratitude et louons ici leur franche et aimable collaboration. Nous remercions les relecteurs qui ont contribué à améliorer la qualité de cet article.

\section{REFERENCES}

Addo P, Quartey M, Abbas M, Adu-Addai B, Owusu E, Okang I, Dodoo A, De Souza D, Ankrah N, Ofori-Adjei D. 2007. Invitro susceptibility of Mycobacterium ulcerans to herbal preparations. Int. J. Trop. Med., 4(2): 1-11. DOI: www.who.int/buruli/events/1.Addo_EN G.pdf?ua /

Adjanohoun E, Cusset G, Issa Lo, Keita A, Lebras M, Lejoly J. 1994. Banque de Données de Médecine Traditionnelle et de Pharmacopée (Pharmel). Notice pour la Collecte et l'Entrée des Données $\left(2^{\text {nd }}\right.$ édn). ACCT : Paris.

Adjet A, Fokou G, Kouame D, Mosi L. 2013. Soins de l'ulcere de Buruli dans le district sanitaire de Yamoussoukro. Conférence InternationaleAfrica 2013 sur l'Ecosanté. https://eujournal.org/index.php/esj/article /viewFile/8682/8394

Adjet AA, Kouame D, Fokou G. 2016. Phytothérapie et lutte contre l'ulcère de Buruli dans le district sanitaire de Yamoussoukro (Cote d'Ivoire) : identification, description, fonction symbolique des plantes et recettes utilisées. Méd. Santé Trop., 26(4): 408413. DOI : $10.1684 / \mathrm{mst} .2016 .0630$ 
Andrade-Cetto A, Heinrich M. 2011. From the field into the lab: useful approaches to selecting species based on local knowledge. Front. Ethnopharm., 2(20): 1-5. DOI: 10.3389/fphar.2011.00 20

Andreoli A, Mou F, Minyem JC, Wantong FG, Djeunga-Noumen, Awah PK, Pluschke G, Um-Boock A, Bratschi MW. 2015. Complete Healing of a Laboratory-Confirmed Buruli Ulcer Lesion after Receiving Only Herbal Household Remedies. PloS Negl. Trop. Dis., $\quad$ 9(11): $\quad$ e0004102. DOI:10.1371/journal.pntd.0004102

Aubry P, Gaüzère B. 2015. Ulcère de Buruli. Med.Trop. Actu. $2015: 1-6 . \quad$ DOI: medecinetropicale.free.fr/cours/ulcere_b uruli.pdf

Betti JL. 2001. Usages traditionnels et vulnérabilité des plantes médicinales dans la réserve de biosphère du Dja, Cameroun. Thèse Doc. $\mathrm{PhD}$, Université Libre de Bruxelles, Bruxelles, p. 202.

Bratschi MW, Bolz M, Minyem JC, Grize L, Wantong FG, Kerber S, Njih Tabah E, Ruf MT, Mou F, Djeunga-Noumen B, Um Boock A, Pluschke G. 2013. Geographic Distribution, Age Pattern and Sites of Lesions in a Cohort of Buruli Ulcer Patients from the Mapé Basin of Cameroon. PLoS Negl. Trop. Dis., 7(6): e2252. DOI: 10.1371/journal.pntd.0002252

Chauty A, Ardant MF, Adeye A, Euverte H, Guédénon A, Johnson C, Aubry J, Nuermberger E, Grosset J. 2007. Promising Clinical Efficacy of Streptomycin-Rifampin Combination for Treatment of Buruli Ulcer (Mycobacterium ulcerans Disease). Antimicrob. Ag. Chem., 51(11): 40294035. DOI:10.1128/AAC.00175-07

Coulibaly B, N'Guessan Kouassi R, Aka N'guetta, Ekaza E, N'Golo DC, Trébissou N, Ouattara L, Bahi C, Coulibaly A, Assandé JM, Mohui P, Yao H, Djaman AJ, Dosso M. 2011. Activité anti-mycobactérienne in vitro des extraits de Phyllanthus amarus (Schum et Thonn) sur les souches de Mycobacterium ulcerans en Côte d'Ivoire. Bull. Soc. Roy. Sc. Liège, $80: 759 \quad-\quad 771 . \quad$ DOI : http://popups.ulg.be/00379565/index.php?id=3257

Diafouka A. 1997. Analyse des usages des plantes médicinales dans quatre régions $\mathrm{du}$ Congo - Brazzaville. Thèse Doc. $\mathrm{PhD}$, Univesité Libre de Bruxelles, Bruxelles, p. 434. Dibong SD, Mpondo Mpondo E, Ngoye A, Kwin MF. 2011. Plantes médicinales utilisées par les populations Bassa de la région de Douala au Cameroun. Int. J. Biol. Chem. Sci., 5(3): 1105-1117. URL: http://ajol.info/index.php/ijbcs

Dibong SD, Mvogo OPB, Vandi D, Ndjib RC, Monkam TF, Mpondo ME. 2015. Ethnobotanique des plantes médicinales anti hémorroïdaires des marchés et villages du Centre et $\mathrm{du}$ Littoral Cameroun. J.Appl.Biosci., 96: 9072 9093. URL; m.elewa.org/Journals/wpcontent/uploads/2015/12/5.dibong.pdf

Dongmo FGI, Enyong OJ, Noumessing MCE, Enyegue MD. 2007. Phytochemical constituents and antioxidant potential of some cameroonian medicinal plants. Pharm., 2: 436-452. URL : pharmacologyonline.silae.it/files/archive s/2007/.../43_Domgo.pdf

Fleurentin J, Weniger B, Bourdy G. 2011. Traditions thérapeutiques et médicine de demain. Les enjeux de l'ethnopharmacologie. Ouest - France : Rennes. ISBN 9782737352546.

Ghourri M, Zidane L, Douira A. 2013. Catalogue des plantes médicinales utilisées dans le traitement de la lithiase rénale dans la province de Tan-Tan (Maroc saharien). Int. J. Biol. Chem. Sci., 7(4): 1688-1700. DOI: http://dx.doi.org/10.4314/ijbcs.v7i4.24

Houndje EMB, Ogni CA, Noudeke N, Farougou S, Youssao AKI, Kpodekon TM. 2016. Recettes ethno-vétérinaire à base de plantes médicinales utilisées pour le traitement de la fièvre aphteuse 
au Bénin. Int. J. Biol. Chem. Sci. 10(5): 2090-2107.

DOI :

http://dx.doi.org/10.4314/ijbcs.v10i5.13

Houngbédji GM, Bouchard P, Frenette J. 2011. Mycobacterium ulcerans infections cause progressive muscle atrophy and dysfunction and mycolactone impairs satellite cell proliferation. Am. J. Physiol. Regulat. Integr. Comp. Physiol., DOI: 10.1152/ajpregu.00393.2010

Johnson RC, Makoutode M, Hougnihin R, Guédénon A, Ifebe D, Boko M, Portaels F. 2004. Le traitement traditionnel de l'ulcère de Buruli au Bénin. Med. Trop., 64: 145-150. URL:www.dermatomag.com/.../145$150 \% 20 \mathrm{Le} \% 20$ traitement $\% 20$ traditionne $1 \% 20 \mathrm{de} \% 201$

Kidik Pouka MC, Ngene JP, Ngoule CC, Mvogo Ottou PB, Ndjib RC, Dibong SD, Mpondo Mpondo E. 2015. Caractérisation des plantes médicinales à flavonoïdes des marchés de Douala (Cameroun). Int. J. Biol. Chem. Sci., 9(3): 1494-1516. DOI : http://dx.doi.org/10.4314/ijbcs.v9i3.32

Landier J, Boisier P, Fotso Piam F, NoumenDjeunga B, Simé J, Wantong FG, Marsollier L, Fontanet A, Eyangoh S. 2011. Adequate wound care and use of bed nets as protective factors against Buruli Ulcer: results from a case control study in Cameroon. PloS Negl. Trop. Dis., 5(11): e1392. DOI: 10.1371/journal.pntd.00001392

Mangambu MJD, Mushagalusa KF, Kadima NJ. 2014. Contribution á l'étude phytochimique de quelques plantes médicinales antidiabétiques de Bukavu, R.D Congo. J. Appl. Biosci., 75:62116220.

URL: www.m.elewa.org/JABS/2014/75/6.pdf

Mpondo ME, Yinyang J, Dibong SD. 2015. Valorisation des plantes médicinales à coumarines des marchés de Douala Est (Cameroun). J. Appl. Biosci., 85:78047823.

DOI : http://dx.doi.org/10.4314/jab.v85i1.6
N'Guessan K., Tra Bi FH, Koné MW. 2009. Étude ethnopharmacologique de plantes antipaludiques utilisées en médecine traditionnelle chez les Abbey et Krobou d'Agboville (Côte d'Ivoire). Ethnopharmacologia, 44: 42-50. URL : www.ethnopharmacologia.org/prelude/p df/bibio-hg-27-guessan.pdf

Owusu-Sekyere E. 2012. Managing the Buruli ulcer morbidity in the Amansie West District of Ghana: can indigenous knowledge succeed? Int. J. Med. Med. Sci., 4:180-185. DOI: 10.5897/IJMMS12.066

Porten K, Sailor K, Comte E, Njikap A, Sobry A, Sihom F, Meva'a Abanda, Eyangoh S, Myatt M, Nackers F, Grais RF. 2009. Prevalence of Buruli Ulcer in Akonolinga Health District, Cameroon: results of a cross sectional survey. PloS Negl. Trop. Dis., 3(6): e466. DOI: 10.1371/journal.pntd.0000466

Pouillot R, Matias G, Wondje CM, Portaels F, Valin N, Ngos F, Njikap A, Marsollier L, Fontanet A, Eyango S. 2007. Risk factors for Buruli Ulcer: A case control study in Cameroon. Plos Negl. Trop. Dis., 1(3): el01. DOI: 10.1371/journal.pntd.0000101

Renzaho AMN, Woods PV, Ackumey MM, Harvey SK, Kotin J. 2007. Communitybased study on knowledge, attitude and practice on the mode of transmission, prevention and treatment of the Buruli ulcer in Ga West District, Ghana. Trop. Med. Int. H., 12(3): 445-458. DOI: 10.1111/j.1365-3156.2006.01795.xSam GH, Adu F, Annan K. 2013. Ethnobotanical survey of medicinal plants used for the treatment of diarrhoea and skin ulcer in the Brong Ahafo region of Ghana. J. Med. Plants Res., 7: 32803285. DOI: 10.5897/JMPR2013.5170

Sarfo FS, Phillips R, Asiedu K, Ampadu E, Bobi N, Adentwe E, Lartey A, Tetteh I, Wansbrough-Jones M. 2010. Clinical Efficacy of Combination of Rifampin and Streptomycin for Treatment of Mycobacterium ulcerans Disease. 
Antimicrob. Ag. Chemoth., 54(9): 36783685. DOI: 10.1128/AAC.00299-10

Seefeld L, Lartey EO, Schindler-Piontek M, Gerhardus A, Razum O. 2013. Herbal Remedies used by BU Patients in the Eastern Region of Ghana. WHO: Geneva.

Tardio J, Pardo-de-Santayana M. 2008. Cultural Importance Indices: A Comparative Analysis Based on the Useful Wild Plants of Southern Cantabria (Northern Spain). Eco. Bot., 62(1): 24-39. DOI: https://doi.org/10.1007/s12231-0079004-5

Tsouh-Fokou PV, Kwadwo Nyarko A, Appiah-Opong R, Tchokouaha Yamthe LR, Addo P, Asante I, Fekam Boyom F. 2015. Ethnopharmacological reports on anti-Buruli ulcer medicinal plants in three West African countries. J. Ethnopharm., 172 (2015): 297-311. DOI:

http://dx.doi.org/10.1016/j.jep.2015.06.0 24

Tsouh-Fokou PV, Abena Adomah KissiTwum, Yeboah-Manu D, Appiah-Opong R, Addo P, Tchokouaha Yamthe LR, Ngoutane Mfopa A, Fekam Boyom F, Kwadwo Nyarko A. 2016. In vitro activity of selected West African mdicinal plants against Mycobacterium ulcerans disease. Molecules, 21: 445. DOI: 10.3390/molecules21040445. Link: www.mdpi.com/journal/molecules.

Yemoa A, Gbenou J, Affolabi D, Moudachirou M, Bigot A, Anagonou S, Portaels F, Leclercq JQ, Martin A. 2011. Buruli ulcer: a review of in vitro tests to screen natural products for activity against Mycobacterium ulcerans. Planta Med., 77: 641-646. DOI : http://dx.doi.org/10.1055/s-00301250642

Yemoa A, Gbenou J, Affolabi D, Moudachirou M, Bigot A, Anagonou S, Portaels F, Martin A, Quetin-Leclercq J. 2015. Beninese medicinal plants as a source of antimycobacterial agents: bioguided fractionation and in vitro activity of alkaloids isolated from Holarrhena floribunda used in traditional treatment of Buruli ulcer. BioMed Res. Int., 2015: 5. DOI: http://dx.doi.org/10.1155/2015/835767

Yemoa AL, Gbenou JD, Johnson RC, Djego JG, Zinsou C, Moudachirou M, QuetinLeclercq J, Bigot A, Portaels F. 2008. Identification et étude phytochimique de plantes utilisées dans le traitement traditionnel de l'ulcère de Buruli au Bénin. Ethnopharmacologia, 42: 48-55. DOI:

http://hdl.handle.net/2078.1/107904

Zahoor M, Yousaf Z, Aqsa T, Haroon M, Saleh N, Aftab A, Javed S, Qadeer M, Ramazan H. 2017. An ethnopharmacological evaluation of Navapind and Shahpur Virkanin district Sheikupura, Pakistan for their herbal Medicines. J. Ethnobiol. Ethnomed., 3:27. DOI: 10.1186/s13002-017-0151-1

Zhang T, Li SY, Converse PJ, Grosset JH, Nuermberger EL. 2013. Rapid, serial, non-invasive assessment of drug efficacy in mice with autoluminescent Mycobacterium ulcerans infection. PLoS Negl. Trop. Dis., 7(12): e2598. DOI : 10.1371/journal.pntd.0002598. 